\title{
Impact of plasma tissue inhibitor of metalloproteinase-1 on long-term survival in patients with gastric cancer
}

\author{
Takaki Yoshikawa, Haruhiko Cho, Akira Tsuburaya, and Osamu Kobayashi \\ Department of Gastrointestinal Surgery, Kanagawa Cancer Center, 1-1-2 Nakao, Asahi-ku, Yokohama 241-0815, Japan
}

\begin{abstract}
Background. The expression of tissue inhibitor of metalloproteinase-1 (TIMP-1) is correlated with tumor invasion and metastases.

Methods. The plasma TIMP-1 concentration was examined preoperatively in 149 patients with gastric cancer who underwent a surgical resection. The cutoff value of TIMP-1 was set at $112.5 \mathrm{ng} / \mathrm{ml}$ based on a previous report. These patients were followed up for more than 5 years prospectively.

Results. Plasma TIMP-1 was positive in 30 of the 149 patients (20.1\%). The overall survival rate was $78.2 \%$ at 5 years in patients with negative plasma TIMP-1, while this rate was $26.7 \%$ at 5 years in patients with positive plasma TIMP-1. By univariate analyses, $T, N, M$, and $R$ category, and TIMP-1, were significant prognosticators. Multivariate analyses demonstrated T, N, and TIMP-1 to be significant prognosticators. The survival curve was clearly separated with respect to TIMP-1.

Conclusion. These results suggest that plasma TIMP-1 is a strong independent prognosticator for the long-term survival of patients with gastric cancer.
\end{abstract}

Key words Gastric cancer - Biomarker - Prognosis · Matrix metalloproteinase $\cdot$ TIMP-1

\section{Introduction}

Gastric cancer is the most frequent malignancy in Japan and the second leading cause of death in the world [1]. Although the mortality has gradually decreased with an increasing incidence in the detection of early-stage disease in Japan, more than $50 \%$ of advanced cases recur, even after a curative resection [2]. The prognosis is extremely poor in patients with recurrent or unresectable gastric cancer [3]. Reliable and convenient bio-

Offprint requests to: T. Yoshikawa

Received: September 9, 2008 / Accepted: November 22, 2008 markers to predict prognosis are needed to optimize surgical and medical management.

Tissue inhibitor of metalloproteinase-1 (TIMP-1) not only inhibits matrix metalloproteinases but also stimulates tumor growth [4]. Nomura et al. [5] and Murray et al. [6] reported enhanced TIMP-1 protein production in gastric tumors. Mimori et al. [7] observed a positive correlation between elevated TIMP-1 mRNA concentrations and tumor progression by analyzing the tumor tissue. In addition, a study conducted by Joo et al. [8] revealed that the intensity of TIMP-1 immunohistochemical staining in stromal cells correlated positively with tumor stage and survival. Recently, elevated TIMP1 protein concentrations in tumor tissue from patients with gastric carcinoma were found to correlate with increased tumor aggressiveness [9].

TIMP-1 was not only detected in various types of tumor tissue but also in the circulating blood of patients with various types of cancer, including ovary [10], colon [11-14], rectum [11-15], lung [16], and breast [17, 18]. In a previous study, we measured plasma TIMP-1 levels in 149 patients with gastric cancer, and this demonstrated a correlation between elevated plasma TIMP-1 and tumor invasiveness and metastasis [19]. Moreover, in patients with gastric cancer in the same cohort, we found that the plasma TIMP-1 concentration also correlated with short-term survival [20]. Although the cutoff values of novel tumor markers are usually evaluated from the value in healthy volunteers, the cutoff value of TIMP-1 was determined based on the TIMP-1 levels in mucosal cancer to evaluate TIMP-1 as a prognostic factor in our previous studies $[19,20]$ and in the present study. In our previous studies, however, the observation period was within 2 years and the results were still preliminary $[19,20]$. Generally, most instances of recurrence have been reported to occur within 2-3 years after surgical resections for gastric cancer [21]. To assess prognostic value, a follow-up period of more than 5 years is recommended. However, the clinical signifi- 
cance of TIMP-1 levels in regard to long-term survival has not been clarified. In the present study, we followed the same cohort of 149 patients for more than 5 years prospectively. The long-term survival results were analyzed to clarify the prognostic value of plasma TIMP-1 levels in patients with gastric cancer.

\section{Patients and methods}

\section{Patients}

Plasma samples were obtained preoperatively from 149 patients with primary gastric adenocarcinoma. None of the patients had undergone any prior treatment. Subsequently, these patients underwent either a total or subtotal gastrectomy at the Kanagawa Cancer Center (Yokohama, Japan) between August 1996 and February 1998. The extent of the tumor was evaluated by the International Union Against Cancer (UICC)/ TNM staging system [22]. Metastases to the liver, lymph nodes, and peritoneum were diagnosed by computed tomography (CT) and then were confirmed during surgery. Metastases to the lung were diagnosed by CT or chest X-ray. Patients who had suspected symptoms of distant metastases were further examined. The patients were followed after surgery for more than 5 years prospectively. The outcomes were evaluated during clinical appointments at the outpatient clinic or by telephone contact.

\section{Measurement of plasma TIMP-1 concentration}

A one-step sandwich enzyme-linked immunosorbent assay (ELISA) kit (Fuji Chemical, Takaoka, Japan) was used to measure the plasma concentration of TIMP-1 [23]. The cutoff value of plasma TIMP-1 was set at $112.5 \mathrm{ng} / \mathrm{ml}$ based on our previous report [20].

\section{Statistical analyses}

A $\chi^{2}$ test was used to compare results the between low and high plasma TIMP-1 groups [24]. The survival curves were calculated using the Kaplan-Meier method and compared using the log-rank test. Cox's proportional hazard model was used to perform univariate and multivariate survival analyses. A $P$ value of less than 0.05 was defined as statistically significant, and the values for data were expressed as means \pm SDs. We used SPSS software (v11.0J Win; SPSS; Chicago, IL, USA) to conduct all statistical tests.

\section{Results}

\section{Clinicopathological factors}

Plasma TIMP-1 was positive in 30 of the 149 patients (20.1\%). When the clinicopathological factors observed in the patients with positive and those with negative plasma TIMP-1 were compared, significant differences were observed in the depth of tumor invasion $(\mathrm{T})$, nodal metastases $(\mathrm{N})$, distant metastases $(\mathrm{M}), \mathrm{R}$ category $(\mathrm{R})$, and the stage of the disease (Table 1).

\section{Survival analyses}

The median follow up period was 63.9 months. The Kaplan-Meier survival curves were significantly different between patients with negative and those with positive plasma TIMP-1 (Fig. 1). The overall survival rate was $78.2 \%$ at 5 years in patients with negative plasma TIMP-1, while the rate was $26.7 \%$ at 5 years in patients with positive plasma TIMP-1. A univariate analysis for overall survival revealed the depth of T, N, M, and the $\mathrm{R}$ category, and plasma TIMP-1, to be statistically significant prognostic factors (Table 2). A multivariate Cox proportional hazards analysis demonstrated that $\mathrm{T}$, $\mathrm{N}$, and plasma TIMP-1 were independent prognostic factors, but $\mathrm{M}$ and $\mathrm{R} 0$ were not (Table 3 ).

When the survival analyses were limited to M0 and R0, the Kaplan-Meier survival curves were significantly different between patients with negative and those with positive plasma TIMP-1 (Fig. 2). The overall survival rate was $84.6 \%$ at 5 years in patients with negative plasma TIMP-1, while the rate was $53.3 \%$ at 5 years in patients with positive plasma TIMP-1. A multivariate analysis demonstrated that $\mathrm{T}$ and plasma TIMP-1 were significant and independent prognosticators, but $\mathrm{N}$ was not (Table 4).

\section{Recurrence patterns (Table 5)}

The initial recurrence site was examined in 127 patients who underwent R0 resection. Any recurrence was observed in 18 of 112 patients with negative TIMP-1 and in 6 of 15 patients with positive TIMP-1. The most frequent initial site was peritoneum in both groups. Recurrence patterns were not different between these two groups $(P=0.377)$.

\section{Discussion}

Our previous report showed a positive correlation between elevated plasma TIMP-1 and tumor invasiveness and metastasis in 149 patients with gastric cancer [19]. Moreover, our next report demonstrated the plasma TIMP-1 concentration to be an independent prognosticator for the short-term survival of patients with gastric carcinoma [20]. In that study, however, the observation period ranged from 350 days to 650 days, which was too short to reach conclusions on the prognostic value. Moreover, 2 patients were excluded from the initial 149 patients due to the very short period of 
Table 1. Background of patients

\begin{tabular}{|c|c|c|c|}
\hline & $\begin{array}{l}\text { TIMP-1-negative } \\
\quad(n=119)\end{array}$ & $\begin{array}{l}\text { TIMP-1-positive } \\
\quad(n=30)\end{array}$ & $P$ value $^{\mathrm{a}}$ \\
\hline Sex (male/female) & $83 / 36$ & 20/10 & 0.744 \\
\hline $\begin{array}{l}\text { Macroscopic type } \\
\text { T1 }\end{array}$ & & & 0.147 \\
\hline Type 0 & 56 & 7 & \\
\hline $\mathrm{T} 2-4$ & & & \\
\hline Type 1 & 4 & 1 & \\
\hline Type 2 & 11 & 3 & \\
\hline Type 3 & 9 & 4 & \\
\hline Type 4 & 11 & 7 & \\
\hline Type 5 & 27 & 8 & \\
\hline Histological type & & & 0.830 \\
\hline Intestinal & 70 & 17 & \\
\hline Diffuse & 49 & 13 & \\
\hline Depth of invasion (T) & & & 0.002 \\
\hline $\mathrm{T} 1$ & 60 & 7 & \\
\hline $\mathrm{T} 2$ & 24 & 3 & \\
\hline T3 & 32 & 17 & \\
\hline $\mathrm{T} 4$ & 3 & 3 & \\
\hline Nodal metastasis $(\mathrm{N})$ & & & $<0.001$ \\
\hline N0 & 70 & 8 & \\
\hline N1 & 30 & 6 & \\
\hline N2 & 10 & 5 & \\
\hline N3 & 9 & 11 & \\
\hline Distant metastasis (M) & & & $<0.001$ \\
\hline M0 & 112 & 19 & \\
\hline M1 & 7 & 11 & \\
\hline R category $(\mathrm{R})$ & & & $<0.001$ \\
\hline R0 & 112 & 15 & \\
\hline R1 & 3 & 3 & \\
\hline $\mathrm{R} 2$ & 4 & 12 & \\
\hline Stage & & & $<0.001$ \\
\hline I & 68 & 8 & \\
\hline II & 20 & 0 & \\
\hline III & 17 & 7 & \\
\hline IV & 14 & 15 & \\
\hline
\end{tabular}

${ }^{a}$ The $\chi^{2}$ method was used to compare the data between the two groups

Overall survival (\%)

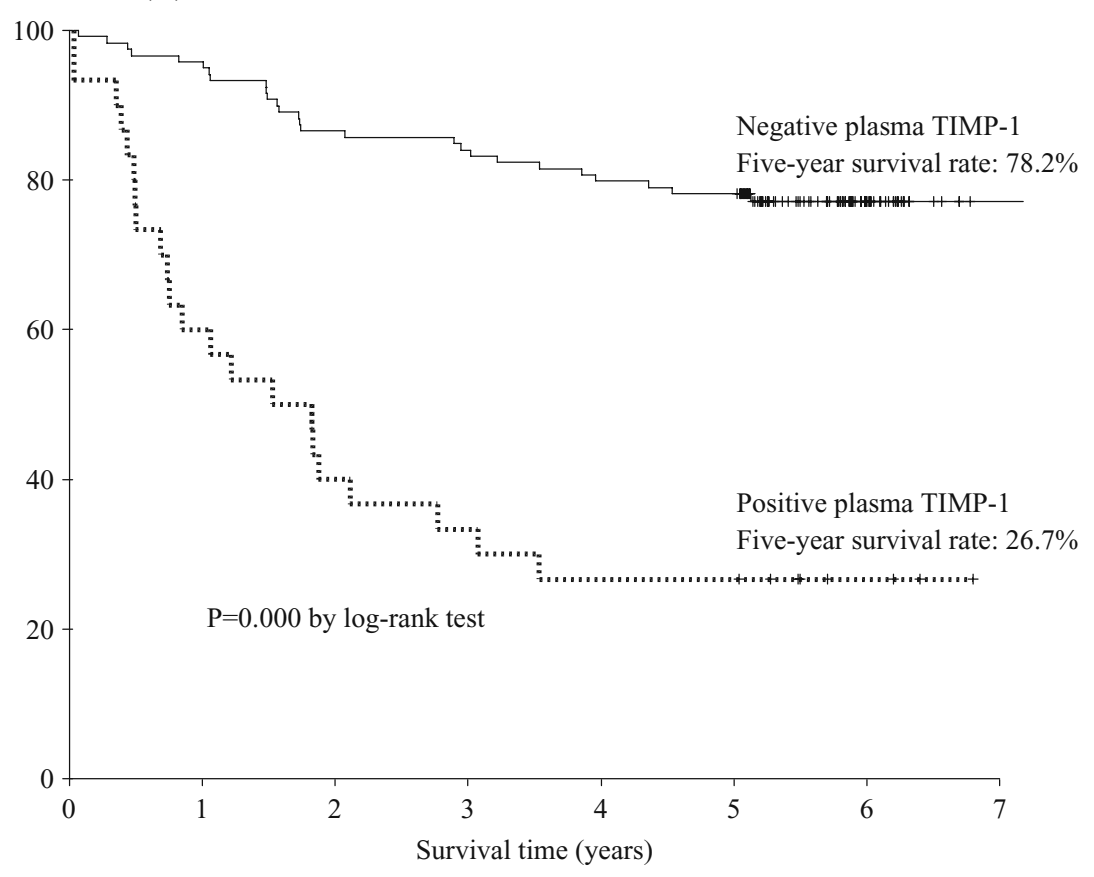

Fig. 1. Overall survival curves in patients with negative (solid line) or positive (broken line) plasma tissue inhibitor of metalloproteinase-1 (TIMP-1). The logrank test revealed a statistically significant difference between the two groups 
Table 2. Univariate Cox proportional hazards analysis for overall survival

\begin{tabular}{|c|c|c|c|}
\hline Factors (category) & Number of patients & $P$ value & Hazard ratio $(95 \% \mathrm{CI})$ \\
\hline \multicolumn{4}{|l|}{ Depth of invasion $(\mathrm{T})$} \\
\hline (T1 vs T2 vs T3 vs T4) & $67,27,49$, and 6 & $<0.001$ & $3.341(2.425-4.604)$ \\
\hline \multicolumn{4}{|l|}{ Lymph node metastasis $(\mathrm{N})$} \\
\hline (N0 vs N1 vs N2 vs N3) & $78,36,15$, and 20 & $<0.001$ & $2.620(2.054-3.341)$ \\
\hline \multicolumn{4}{|l|}{ Distant metastasis $(\mathrm{M})$} \\
\hline (M0 vs M1) & 131 and 18 & $<0.001$ & $12.082(6.374-22.900)$ \\
\hline \multicolumn{4}{|l|}{$\mathrm{R}$ category $(\mathrm{R})$} \\
\hline (R0 vs R1 vs R2) & 127,6 , and 16 & $<0.001$ & $5.216(3.633-7.490)$ \\
\hline TIMP-1 (negative vs positive) & 119 and 30 & $<0.001$ & $5.566(3.146-9.849)$ \\
\hline
\end{tabular}

Table 3. Multivariate Cox proportional hazards analysis for overall survival

\begin{tabular}{|c|c|c|c|}
\hline Factors (category) & Number of patients & $P$ value & Hazard ratio $(95 \% \mathrm{CI})$ \\
\hline \multicolumn{4}{|l|}{ Depth of invasion (T) } \\
\hline (T1 vs T2 vs T3 vs T4) & $67,27,49$, and 6 & $<0.001$ & $2.026(1.369-2.999)$ \\
\hline \multicolumn{4}{|l|}{ Lymph node metastasis $(\mathrm{N})$} \\
\hline (N0 vs N1 vs N2 vs N3) & $78,36,15$, and 20 & 0.001 & $1.662(1.235-2.236)$ \\
\hline \multicolumn{4}{|l|}{ Distant metastasis (M) } \\
\hline (M0 vs M1) & 131 and 18 & 0.732 & $0.859(0.360-2.048)$ \\
\hline \multicolumn{4}{|l|}{$\mathrm{R}$ category $(\mathrm{R})$} \\
\hline (R0 vs R1 vs R2) & 127,6 , and 16 & 0.101 & $1.884(0.884-4.014)$ \\
\hline TIMP-1 (negative vs positive) & 119 and 30 & 0.005 & $2.285(1.277-4.091)$ \\
\hline
\end{tabular}

Table 4. Multivariate Cox proportional hazards analyses for overall survival when limited to M0 and R0

\begin{tabular}{lllr}
\hline Factors (category) & Number of patients & $P$ value & Hazard ratio (95\% CI) \\
\hline $\begin{array}{l}\text { Depth of invasion (T) } \\
\quad(\text { T1 vs T2 vs T3 vs T4) }\end{array}$ & $67,26,30$, and 2 & $<0.001$ & $2.713(1.642-4.482)$ \\
$\begin{array}{l}\text { Lymph node metastasis (N) } \\
(\text { N0 vs N1 vs N2 vs N3) }\end{array}$ & $78,31,11$, and 5 & 0.218 & $1.336(0.843-2.120)$ \\
TIMP-1 (negative vs positive) & 110 and 15 & 0.013 & $3.166(1.269-7.897)$ \\
\hline
\end{tabular}

Overall survival (\%)

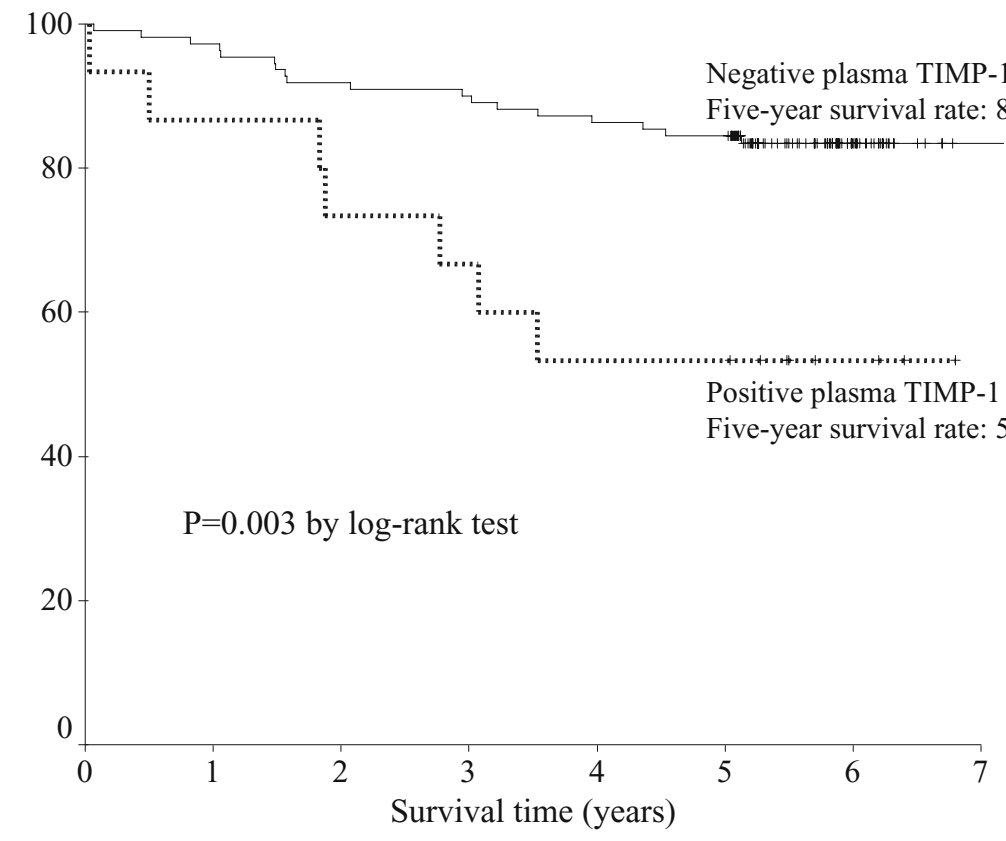

Fig. 2. Overall survival curves in $\mathrm{M} 0$ and R0 patients with negative (solid line) or positive (broken line) plasma TIMP-1. The log-rank test revealed a statistically significant difference between the two groups 
Table 5. Recurrence patterns in 127 patients who underwent $\mathrm{R} 0$ resection

\begin{tabular}{lcc}
\hline & $\begin{array}{c}\text { TIMP-1-negative } \\
(n=112)\end{array}$ & $\begin{array}{c}\text { TIMP-1-positive } \\
(n=15)\end{array}$ \\
\hline Any recurrence & 18 & 6 \\
Initial site & 5 & 0 \\
$\quad$ Hematogenous & 4 & 2 \\
Lymph node & 14 & 5 \\
$\quad$ Peritoneum & & \\
\hline
\end{tabular}

Some patients had initial recurrence in more than one site

the follow up, and the remaining 147 patients were analyzed. In addition, the disease stage had been evaluated by the Japanese classification of gastric carcinoma (JCGC) of the Japanese Research Society for Gastric Cancer [25], which defines nodal metastasis by the location of metastatic nodes. In the present study, all of the patients were followed for more than 5 years and tumor spread was evaluated using the UICC/TNM, which reflects survival more accurately than the JCGC [26]. The JCGC, UICC/TNM defines nodal metastasis by the number of metastatic nodes. Plasma TIMP-1 concentrations were found to be correlated with serosal invasion, metastases, and advanced stages of the disease [19]. This suggests the plasma TIMP- 1 concentration to be a prognosticator for long-term survival. In the present study, the cutoff value for TIMP-1 was the same as that used in our previous study [19], in which the cutoff value had been determined by the TIMP- 1 value in mucosal cancer. Mucosal cancer or noninvasive carcinoma does not initiate the degradation processes in which matrix metalloproteinases may play important roles. With this cutoff value, the concentration of plasma TIMP-1 was a significant factor for long-term survival, based on both univariate and multivariate analyses, suggesting that the concentration of plasma TIMP-1 is as strong an independent prognosticator as $\mathrm{T}, \mathrm{N}$, and the $\mathrm{R}$ category. However, the validity of the plasma TIMP-1 concentration using this cutoff value should be confirmed in an independent cohort of patients in a future study.

Generally, the most important factors affecting survival were $\mathrm{T}, \mathrm{N}, \mathrm{M}$, and the $\mathrm{R}$ category. In the present study, multivariate analysis revealed that $\mathrm{T}, \mathrm{N}$, and plasma TIMP-1 expression were statistically significant indicators for survival; the $\mathrm{M}$ factor and $\mathrm{R}$ category were not independent factors in this study. When the survival analyses were limited to $\mathrm{M} 0$ and $\mathrm{R} 0, \mathrm{~T}$ and plasma TIMP-1 were still independent prognostic factors, based on a multivariate analysis. Considering the value of the prognosticators, plasma TIMP-1 expression was found to be superior to $\mathrm{N}$ in terms of the $P$ value and hazard ratio and plasma TIMP-1 expression was superior to $\mathrm{T}$ in terms of the hazard ratio in this analysis. These results indicate that plasma TIMP-1 concentration is a promising and powerful independent prognostic factor in patients with gastric cancer, in comparison to the conventional factors such as T, N, M, and the $\mathrm{R}$ category.

The prognostic significance of TIMP-1 levels in the circulating blood has been examined in several types of malignancy [10-16]. The prognostic value of TIMP-1 was confirmed in cancer of the ovary by Manenti et al. [10], in cancer of the lung by Ylisirnio et al. [16], and in cancer of the breast by Kuvaja et al. [18], while the results were controversial for colorectal cancer [11-15]. Holten-Andersen et al. [11, 15] and Yukawa et al. [14] showed the prognostic significance of plasma concentrations of TIMP- 1 in patients with colorectal cancer. Ishida et al. [13] and Oberg et al. [12] measured serum samples and demonstrated no significant correlation between TIMP-1 levels and survival. In another report, Holten-Anderson et al. [27] examined serum and plasma samples for the measurement of TIMP-1 and concluded that serum was not suitable because the serum TIMP-1 was affected by repeated freezing and thawing. Plasma samples may be required to evaluate the clinical significance of TIMP-1 accurately.

Previously, we measured TIMP-1 proteins in gastric cancer tissues quantitatively and found that intratumoral TIMP-1 protein was elevated in more aggressive tumors and was a significant factor for survival [28]. Murray et al. [6] examined TIMP-1 expression in gastric cancer by immunohistochemistry. They found that TIMP-1 expression was confined to tumor cells [6]. On the other hand, Joo et al. [8] reported that TIMP-1 expression was detected predominantly in the peritumoral stromal cells rather than tumor cells. Thus, TIMP1 protein is produced both in tumor and stromal cells and then flows into the bloodstream.

In the present study, prognostic significance was observed not only in the analysis of all patients but also in the analysis of M0 and R0. Moreover, the plasma TIMP-1 expression clearly separated patients with a good and a poor prognosis, regardless of the nodal status and the stage, thus suggesting that plasma TIMP1 could be a prognosticator not only for early disease but also for advanced disease. In other words, even patients classified as in the same stage include subgroups with a different risk for survival, which was clearly separated by the plasma TIMP-1 concentration. HoltenAnderson et al. [11] also showed that survival was associated with the concentration of plasma TIMP-1 in Dukes' stage B in patients with colorectal cancer. Therefore, the plasma TIMP-1 concentration may be used to separate patients with good and poor prognoses.

Among the prognostic factors, the plasma level of TIMP-1 has a great advantage in comparison to other prognosticators such as $\mathrm{T}, \mathrm{N}$, and $\mathrm{M}$ because it requires a far less invasive procedure to yield useful information. $\mathrm{T}$ and $\mathrm{N}$ are predicted by clinical examinations such as 
endoscopy, endoscopic ultrasonography, and CT, and must be confirmed by the pathological examination of resected specimens. $\mathrm{M}$ is evaluated by $\mathrm{CT}$, laparoscopy, and laparotomy. On the other hand, only a blood sample is required for the measurement of plasma TIMP-1 concentrations. Moreover, the ELISA technique is convenient, reproducible, and reliable. Therefore, plasma TIMP-1 concentration could provide useful prognostic information by a simple and less invasive method in patients with gastric cancer.

Recurrence patterns were not different when stratified by TIMP-1 positivity. This suggests that TIMP-1 may not be a marker for specific recurrence patterns, although the number of recurrences was too small to reach a definite conclusion.

In conclusion, the results of the present study suggested that plasma TIMP-1 expression was a significant strong independent prognosticator for the survival of patients with gastric cancer.

Acknowledgments This work was supported in part by a Grant-in Aid for Cancer Research (10-27) from the Ministry of Health, Labour and Welfare of Japan.

\section{References}

1. Torrado J, Santiseban A, Ruiz-Ederra J. Genetic susceptibility to gastric cancer. Hepatogastroenterology 2001;48:1544-7.

2. Adachi Y, Kitano S, Sugimachi K. Surgery for gastric cancer: 10year experience worldwide. Gastric Cancer 2001;4:166-74.

3. Ohtsu A, Yoshida S, Saijo N. Disparities in gastric cancer chemotherapy between the east and west. J Clin Oncol 2006;10: 2188-93.

4. Gomez DE, Alonso DF, Yoshiji H, Thorgeirsson UP. Tissue inhibitors of metalloproteinases: structure, regulation and biological functions. Eur J Cell Biol 1999;74:111-22.

5. Nomura H, Fujimoto N, Seiki M, Mai M, Okada Y. Enhanced production of matrix metalloproteinases and activation of matrix metalloproteinase 2 (gelatinase A) in human gastric carcinomas. Int J Cancer 1996;69:9-16.

6. Murray GI, Duncan ME, Arbuckle E, Melvin WT, Fothergill JE. Matrix metalloproteinases and their inhibitors in gastric cancer. Gut 1998:43:791-7.

7. Mimori K, Mori M, Shiraishi T, Fujie T, Baba K, Haraguchi M, et al. Clinical significance of tissue inhibitor of metalloproteinase expression in gastric carcinoma. Br J Cancer 1997;76:531-6.

8. Joo YE, Seo KS, Kim HS, Rew JS, Park CS, Kim SJ. Expression of tissue inhibitors of metalloproteinases (TIMPs) in gastric cancer. Dig Dis Sci 2000;45:114-21.

9. Yoshikawa T, Tsuburaya A, Kobayashi O, Sairenji M, Motohashi $\mathrm{H}$, Yanoma S, et al. Intratumoral concentrations of tissue inhibitor of matrix metalloproteinase-1 in patients with gastric cancer. A new biomarker for invasion and its impact on survival. Cancer 2001;91:1739-44.

10. Manenti L, Paganoni P, Floriani I, Landoni F, Torri V, Buda A, et al. Expression levels of vascular endothelial growth factor, matrix metalloproteinases 2 and 9 and tissue inhibitor of metalloproteinases 1 and 2 in the plasma of patients with ovarian carcinoma. Eur J Cancer 2003;39:1948-56.

11. Holten-Andersen MN, Stephens RW, Nielsen HJ, Murphy G, Christensen JJ, Steler-Stevenson W, et al. High preoperative plasma tissue inhibitor of metalloproteinase-1 levels are associ- ated with short survival of patients with colorectal cancer. Clin Cancer Res 2000;6:4292-9.

12. Oberg A, Hoyhtya M, Tavelin B, Stenling R, Lindmark G. Limited value of preoperative serum analyses of matrix metalloproteinases (MMP-2, MMP-9) and tissue inhibitors of matrix metalloproteinases (TIMP-1, TIMP-2) in colorectal cancer. Anticancer Res 2000;20:1085-91.

13. Ishida H, Murata N, Hayashi Y, Tada M, Hashimoto D. Serum levels of tissue inhibitor of metalloproteinases-1 (TIMP-1) in colorectal cancer patients. Surg Today 2003;33:885-92.

14. Yukawa N, Yoshikawa T, Akaike M, Sugimasa Y, Rino Y, Masuda M, et al. Impact of plasma tissue inhibitor of matrix metalloproteinase-1 on long-term survival in patients with colorectal cancer. Oncology 2007;72:205-8.

15. Holten-Andersen M, Christensen IJ, Nilbert M, Bendahl PO, Nielsen HJ, Brunner N, et al. EORTC-Receptor and Biomarker Group. Association between preoperative plasma levels of tissue inhibitor of metalloproteinases 1 and rectal cancer patient survival. A validation study. Eur J Cancer 2004;40:64-72.

16. Ylisirnio S, Hoyhtya M, Makitaro R, Paaakko P, Kinnula VL, Turpeenniemi-Hujanen T, et al. Elevated serum levels of type I collagen degradation marker ICTP and tissue inhibitor of metalloproteinase (TIMP) 1 are associated with poor prognosis in lung cancer. Clin Cancer Res 2001;7:1633-7.

17. Schrohl AS, Mueller V, Christensen IJ, Pantel K, Thomssen C, Brunner N. A comparative study of tissue inhibitor of metalloproteinases-1 levels in plasma and tumour tissue from patients with primary breast cancer and in plasma from patients with metastatic breast cancer. Tumour Biol 2008;29:181-7.

18. Kuvaja P, Talvensaari-Mattila A, Turpeenniemi-Hujanen $T$. High preoperative plasma TIMP-1 is prognostic for early relapse in primary breast carcinoma. Int J Cancer 2008;123:84651.

19. Yoshikawa T, Saitoh M, Tsuburaya A, Kobayashi O, Sairenji M, Motohashi $\mathrm{H}$, et al. Tissue inhibitor of matrix metalloproteinase-1 in the plasma of patients with gastric carcinoma. A possible marker for serosal invasion and metastasis. Cancer 1999;86: 1929-35.

20. Yoshikawa T, Tsuburaya A, Kobayashi O, Sairenji M, Motohashi $\mathrm{H}$, Yanoma S, et al. Prognostic value of tissue inhibitor of matrix metalloproteinase- 1 in plasma of patients with gastric cancer. Cancer Lett 2000;151:81-6.

21. Sakar B, Karagol H, Gumus M, Basaran M, Kaytan E, Argon A, et al. Timing of death from tumor recurrence after curative gastrectomy for gastric cancer. Am J Clin Oncol 2004;27:2059.

22. Sobin LH, Wittekind Ch. TNM classification of malignant tumors. 6th ed. New York: Wiley-Liss; 2002.

23. Hayakawa T, Yamashita K, Kodama S, Iwata H, Iwata K. Tissue inhibitor of metalloproteinases and collagenase activity in synovial fluid of human rheumatoid arthritis. Biomed Res 1991;12: $169-73$.

24. Kodama S, Iwata K, Iwata H, Yamashita K, Hayakawa T. Rapid one-step sandwich enzyme immunoassay for tisssue inhibitor of metalloproteinases. J Immunol Methods 1990;127:103-8.

25. Japanese Research Society for Gastric Cancer. Japanese classification of gastric carcinoma. First English ed. Tokyo: Kanehara; 1995.

26. Sayegh ME, Sano T, Dexter S, Katai H, Fukagawa T, Sasako M. TNM and Japanese staging systems for gastric cancer: how do they coexist? Gastric Cancer 2004;7:140-8.

27. Holten-Andersen MN, Schrohl AS, Brunner N, Nielsen HJ, Hogdall CK, Hogdall EV. Evaluation of sample handling in relation to levels of tissue inhibitor of metalloproteinases-1 measured in blood by immunoassay. Int J Biol Markers 2003;18: 170-6.

28. Yoshikawa T, Tsuburaya A, Kobayashi O, Sairenji M, Miyagi Y. Protein levels of tissue inhibitor of metalloproteinase-1 in tumor extracts as a marker for prognosis and recurrence in patients with gastric cancer. Gastric Cancer 2006;9:106-13. 